\title{
ENFOQUES SOBRE LA FILOSOFÍA DE RODOLFO KUSCH. EL MÉTODO, LO POPULAR Y EL INDÍGENA COMO HORIZONTES DE PREGUNTA EN LA FILOSOFÍA AMERICANA ${ }^{l}$ \\ Perspectives on Rodolfo Kusch's philosophy: method, popular approach and indigenous people as questioning horizons in Latin American philosophy
}

Alejandro Viveros Espinosa*

Resumen

El artículo recorre la obra de Rodolfo Kusch posicionando sus principales propuestas en la construcción de tres enfoques convergentes en su filosofía. El primer enfoque está relacionado con la fenomenología y la cultura. El segundo enfoque se refiere a la influencia de la antropología y el cuestionamiento por el símbolo. El tercer enfoque despliega una aproximación filosóficopolítica. Estos enfoques permiten introducir tres "horizontes de pregunta" principalmente relacionados con el método, con lo popular y con lo indígena, que son expuestos como asuntos centrales en toda su obra. Estos "horizontes de pregunta" buscan comprender los alcances de la filosofía de Kusch y su contribución al pensamiento filosófico americano.

Palabras clave: Rodolfo Kusch, Filosofía Americana, Método, Popular, Indígena.

\section{Abstract}

This paper analyses the work of Rodolfo Kusch remarking his main philosophical proposals through three correlated perspectives. The first perspective refers to phenomenology and culture. The second focuses on the influence of anthropology and the problem of symbols. The third posits a political-philosophical approximation. These perspectives operate interdependently as the background for Kusch's philosophy and allow us to introduce three "questioning horizons" related to the method, the popular and the indigenous as the central issues of his philosophical work. Thus, these "questioning horizons" build a platform to understand Kusch's philosophy and his contribution to the Latin American philosophy.

Key words: Rodolfo Kusch, Latin American Philosophy, Method, Popular, Indigenous.

Una América que deje de ser un escenario, para convertirse en la tierra nutricia donde brota nuestra vida. Una América así nos hace falta para reasumir nuestra humanidad y nuestra solidez, como para empezar de nuevo, como si descendiéramos al infierno. ¿Para qué? Pues para hacer ver que no hay tal caos, ni tal infierno. Que el infierno mismo se da en la vida (Kusch, América Parda 4, 188).

\footnotetext{
${ }^{1}$ Este artículo es resultado del proyecto Conicyt/Fondecyt Postdoctorado № 3140318 "Construcciones identitarias y traducciones culturales. Reflexiones filosófico-políticas sobre la noción de indio en Mesoamérica y los Andes coloniales (1570-1640)".
} 


\section{ACLARACIONES INICIALES}

Pensar acerca de la obra de Gunther Rodolfo Kusch (1922-1979) nos encamina hacia un reposicionamiento de diferentes influencias y perspectivas dentro de las cuales $s u$ pensamiento toma lugar. Queremos iniciar este ensayo propedéuticamente. Para ello indicaremos algunos de los múltiples trabajos realizados respecto del pensamiento de Kusch. Intentaremos conducir algunas aclaraciones iniciales de la recepción e interpretación que ha logrado su filosofía en diferentes períodos.

Pues bien, las referencias bibliográficas de su obra no son escasas. Durante la década de 1970 recalcamos los textos de Casalla (1973) y Cullen (1978) como antecedentes inaugurales en el reconocimiento de la obra de Kusch. En ambos textos se evidencia un carácter propositivo respecto de las posibilidades de una filosofía de la cultura en un horizonte latinoamericano.

Durante la década de 1980 identificamos las lecturas de Scannone (1984), Toribio (1984), Reigadas (1986) y Azcuy (1989). Scannone reconoce en Kusch el problema de la sabiduría popular y lo simbólico como un asunto fundamental para la filosofia latinoamericana. Toribio se focaliza en la lógica de la negación como parte fundamental del método de Kusch. Reigadas remarca la contribución de Kusch en el cuestionamiento por una antropología americana. Subrayamos el libro de Azcuy debido a su carácter compilatorio - de varios autores y perspectivas- y porque marca el reconocimiento de la intelectualidad argentina hacia la obra de Kusch. Destacamos las Cuartas Jornadas de Pensamiento Argentino realizadas en 1989 y dedicadas al pensamiento de Kusch.

De esas Jornadas, ya en la década de 1990, se editan sus Actas, proyecto a cargo de Camaly, Jalfen y Langón (1993). Este breve texto despliega el pensamiento de Kusch mediante problemas teóricos, principalmente referidos a la construcción de una ontología americana. Encontramos también, durante este período, los textos de Steffen (1990), Flores (1991), Montes (1995), Sada (1996) y Bordas de Rojas (1997). Steffen direcciona los planteamientos de la filosofia de Kusch en términos existenciales e identitarios hacia una perspectiva psicológica acerca de lo sudamericano. Flores refiere comparativamente a la noción de mito destacando los enlaces filosófico-literarios en su obra. Montes retoma la filosofía de Kusch a partir de una revisión de sus propuestas respecto del hombre americano. Sada, por su parte, despliega un análisis hacia la idea de filosofia en Kusch. Finalmente, Bordas de Rojas sostiene un acercamiento "a la intemperie" que profundiza en la obra de Kusch recuperando sus reflexiones en el horizonte de una fundamentación ontológica americana. Asimismo, acentuamos la investigación monográfica de Pagano (1999), probablemente el trabajo más completo -hasta la fecha- de descripción filosófica y recopilación bibliográfica. El escrito de Pagano desarrolla un detallado trabajo de análisis referido a la vida y la obra de Kusch que considera sus aportes y proyecciones frente al problema de lo intercultural.

Ya en los 2000 identificamos el revitalizador posicionamiento conceptual de la obra de Kusch presente en el texto de Rubinelli (2001) y la sugerente aproximación 
filosófico-literaria de Maturo (2006). Así también, destacamos el texto de la Unión del Personal Civil de la Nación (2006) titulado Octubre Nacional donde Kusch es interpretado dentro del ideario peronista contemporáneo de izquierda. No podemos dejar de lado la publicación de sus Obras Completas (2009) -proyecto elaborado entre 1998 y 2003 por la editorial Fundación Ross-, puesto que las utilizaremos como referencia durante este escrito.

Remarcamos la continuidad de esta lectura sobre Kusch con el libro de Fraschini (2010) donde se posiciona a su pensamiento como fundamento para la reformulación del proyecto político y cultural argentino. Por otra parte, resaltamos el breve ensayo de Cullen (2010) donde se recupera el alcance y los aportes de Kusch en la filosofía latinoamericana contemporánea. Señalamos también el número 77 de Análisis. Revista Colombiana de Humanidades de la Universidad Santo Tomás (2010). Este número, dedicado a su obra, releva aspectos centrales de sus propuestas acerca de la filosofía y la cultura latinoamericana. Finalmente, debemos incluir la traducción al inglés de Kusch (2010) titulada Indigenous and Popular Thinking in América. Este texto, publicado por la Universidad de Duke en EE.UU., presenta la incorporación y divulgación de sus propuestas filosóficas desde la teoría decolonial ${ }^{2}$.

Sin embargo, nuestro escrito sigue un camino alternativo respecto de estas corrientes. Buscamos pensar con Kusch y reconocer cómo toma lugar su pensamiento. Indudablemente, pensar con Kusch implica también -en muchos sentidos-dejarlo hablar. Dejar hablar a sus textos implica a su vez posicionar ciertos acercamientos que nos ayuden a reconocer los asuntos centrales de su filosofia y que, de forma propositiva, nos permiten volver a indagar, volver a preguntar, por su filosofia. Adentrar el pensamiento de Kusch no es un trabajo sencillo. Intentaremos priorizar sus particularidades respondiendo tanto a las influencias que mantuvo con diferentes filósofos y pensadores (principalmente europeos) en busca de una apertura hacia sus propios horizontes de pregunta ${ }^{3}$. Bajo

\footnotetext{
${ }^{2}$ Considerando las interpretaciones ya expuestas respecto de la obra de Kusch, queremos subrayar su influencia en la teoría decolonial. Esta corriente mantiene una cercanía con el pensamiento de Kusch en la medida en que se nutre de sus ideas. Su participación en este proyecto filosófico contribuye, principalmente, a la relectura y el rescate del método, lo popular y lo indígena como fuentes o plataformas en la construcción de un pensamiento filosófico no eurocéntrico situado en América. En este contexto, las propuestas de Kusch funcionan como apuntalamientos teóricos en la ruptura epistémica con la tradición filosófica occidental. Podemos reconocer su impacto en la teoría decolonial por medio del concepto de "pensamiento fronterizo" (border thinking) -acuñado por Mignolo-, donde Kusch muestra cómo "pensar desde las ruinas de las categorías amerindias" (Mignolo, Local histories / Global designs, 149-164). La teoría decolonial posiciona a Kusch mediante el concepto de "conciencia de inmigrante" (inmigrant consciousness), rescatando su ruptura epistémica y ontológica. Mignolo define la "conciencia de inmigrante" como "una existencia fuera de lugar", lo que permite a la perspectiva decolonial incorporar el cuestionamiento kuscheano sobre el "habitar americano" como fundamento para una crítica a la modernidad occidental (Mignolo, Introduction, XV-LIV). Desde ahí la teoría decolonial ha interpretado su obra como un antecedente importante y fundacional, incluyéndolo en su canon filosófico (Dussel, Mendieta y Bohórquez).

${ }^{3}$ Por horizonte de pregunta queremos retomar, especialmente, la comprensión hecha por la fenomenología. La noción de horizonte, siguiendo a Husserl, nos permite incorporar un enlace entre la expectativa y la experiencia, porque "toda experiencia tiene un horizonte experiencial" en tanto una "estructura" que está referida al mundo de
} 
palabras y símbolos -muchas veces crípticos- su pensamiento va encontrando diversas formas de explicarse, de ejemplificarse. El estilo de su escritura es provocador. Provoca al lector presentando y yuxtaponiendo problemas de corte ontológico, axiológico, político e identitario en el marco del cuestionamiento fundamental respecto de un pensamiento filosófico propiamente americano. Kusch es un librepensador que vuelve a lo americano desde la tradición europea en una relación de diálogo y tensión con ella.

Kusch nos obliga a re-articular nuestros prejuicios y a recoger de estos algunos elementos particularmente soslayados. Luego, su cuestionamiento concerniente a la "esencialidad de la cultura americana" contempla aquello que esta ha negado, ocultado, ahuyentado (Kusch, La seducción, 17-60). Esta actitud de sospecha conduce el objeto de este escrito, particularmente relacionado con el desarrollo de tres perspectivas fundamentales, es decir, tres enfoques desde los cuales sea posible adentrar el método, lo popular y lo indígena de su filosofía. Por ello, es conveniente introducir el pensamiento de Kusch a la luz de un horizonte epocal o bien un contexto histórico-cultural latinoamericano muy particular ${ }^{4}$. En este punto, el horizonte epocal nos muestra cómo Kusch intenta responder una pregunta identitaria y existencial, que se formula en toda América y que mantiene una importante resonancia ${ }^{5}$. En su obra encontramos una propositiva lectura de América, principalmente en su relación con aquello que nombra como el "pensamiento filosófico indígena". Algo de ello insinúa Kusch en un breve texto titulado Sobre el cursillo de filosofia indígena:

la vida como un "margen o estructura co-presente", constituyendo un "mundo de asuntos, de co-datos" (Husserl, Erfahrung und Urteil, 8-9, 26-38). Para Heidegger la temporalidad funciona como horizonte de cualquier comprensión del ser, donde horizonte equivale a los límites en el marco de la pregunta tanto por el sentido y la existencia (Heidegger, Ser y Tiempo, 5, 25-29) como por la temporalidad (historicidad) y la trascendencia del mundo, comprendiendo al concepto de horizonte como unidad extática de la temporalidad (69, 379-396). La noción de horizonte de pregunta busca fortalecer el alcance de la noción de horizonte como el límite de la totalidad de las cosas dadas, y a la vez como lo que las constituye en cuanto tales (Kuhn, 106-123). Incorporamos a ello la lectura de Ottmar Ette respecto del horizonte de pregunta (Fragehorizont) como un cuestionamiento relativo a la experiencia (Erfahrung) y también a la vivencia (Erlebnis). El horizonte de pregunta cuestiona y pesquisa, en consecuencia, las posibilidades de análisis, comprensión e interpretación, en este caso referidas a los propios problemas y límites en el pensamiento filosófico de Rodolfo Kusch.

${ }^{4}$ De forma panorámica podemos indicar que el siglo XX latinoamericano y particularmente la realidad argentina responden a un modelo desarrollista e industrializador (Halperin Donghi; Carmagnani). Brevemente queremos señalar que Kusch se encuentra en medio de estos procesos "modernizadores" acelerados que acarrearon complejos cambios sociales, políticos y económicos.

${ }^{5}$ La pregunta por la identidad durante el siglo XX latinoamericano encierra múltiples variantes. Podríamos establecer por lo menos dos grandes conceptualizaciones, a saber, la noción de mestizaje y la noción de subalterno (con diferentes variaciones políticas, étnicas y de género). Ambas variantes reconocen en lo latinoamericano un problema identitario abierto. Ciertamente el concepto de mestizaje tiene una mayor presencia en el pensamiento latinoamericano principalmente desde Vasconcelos hasta Echeverría. Sin embargo, la comprensión de lo subalterno también es parte de la reflexión contemporánea y ha sido recogida por lecturas latinoamericanas de diversa raigambre. Destacamos el acercamiento desde la indianidad y la subalternidad (Rivera y Barragán). Ahora bien, Kusch parece deambular entre un mestizaje cultural en una perspectiva ontológica y una lectura subalterna, ulteriormente, identificada con lo popular y lo indígena, que funcionan como plataformas para el cuestionamiento identitario latinoamericano. 
Mi intención es la de crear una especie de mística de ser sudamericanos. Para ello tenemos que sujetarnos aquí y ahora y evitar en todo sentido las modas literarias o filosóficas y enfrentar nuestra desnuda condición de haber nacido en América. Tengo fe en que de esta manera, al fin, habremos de resolver con sencillez nuestros problemas. Se trata de llevar a América a la conciencia, para ello nada mejor que el pensamiento indígena. Creo, por otra parte, y esto entra en la parte fundamental de mi cursillo, todos llevamos al indígena adentro aunque vengamos de Buenos Aires, de Nueva York o de Berlín $(1,307)$.

Este punto es muy interesante pues conduce la noción de lo indígena como una vía para resolver problemas existenciales e identitarios relativos a la condición americana. Esta resolución implica concientizar a América por medio del ejercicio de un pensamiento indígena como una idea que "todos llevamos adentro". Kusch es siempre críptico y nos lleva a cuestionar a lo indígena como el trasfondo de aquella esencialidad americana. Por ello, al hablar de un "pensamiento filosófico indígena" o bien de lo indígena, Kusch lo presenta - a lo largo de su obra- bajo la noción de lo "arcaico o antiguo", o bien como "pensamiento seminal" (Kusch, El pensamiento 2, 335-490). En ambas nociones persiste la relación con una conducta o modo de vida que releva una comprensión particular de la existencia. La noción de lo seminal es clara en este aspecto, pues despliega la contraposición entre dos modos de pensar y vivir, uno occidental, denominado "causal", y otro indígena, identificado como "seminal". Lo seminal, lo arcaico y lo antiguo se contrastan con lo causal, lo moderno y lo actual. La afección de lo seminal (o bien de lo arcaico) se opone cualitativamente a la inteligencia causal, moderna, inquisitiva y descriptiva. Indica Kusch en El pensamiento indígena y popular en América refiriendo al pensamiento causal que:

Se reduce a un riguroso solucionismo, consistente este en un credo sobre la modificación de las partes, regido por un criterio analítico, cuantitativo y causal, respaldado, a su vez, por la urgencia de un quehacer constante $(2,476)$.

Frente al modo de pensar causal Kusch propone y contrapone el pensamiento seminal. El término seminal remite al latín semen (semilla) y hace referencia al "origen", al "germen", a la "fuente", a aquello que "se ve crecer desde la tierra" (VOX, 2001). En el pensamiento seminal predomina, siguiendo a Kusch, un "sujeto que siente el favor o disfavor del mundo" (El pensamiento 2, 473), que se pregunta ya no por el porqué, sino por el cómo de la existencia. Kusch comprende al pensamiento seminal como "un pensar por entrancias, que personaliza al mundo y destaca la globalidad de este, porque enfrenta el desgarramiento original entre lo favorable y lo desfavorable y requiere obsesivamente la unidad llevada por un afán de salvación" (477). El fondo de este asunto estriba en encontrar una manera de cruzar el límite entre dos modos de pensar a fin de responder "¿de qué manera, especialmente en el ciudadano, se da junto al pensar causal, otro pensar al modo indígena?" (479). 
Desde esta perspectiva podemos a su vez destacar un rasgo característico en su pensamiento, relacionado mayormente con su método y extensivo en toda su obra. Precisamente, si abordamos la contraposición entre el pensar causal y el pensar seminal. Para ello, posicionamos como enlace al concepto de "dialéctica" en tanto que "juego de los contrarios". La palabra "dialéctica" como herramienta conceptual está presente en varios momentos de su obra. Esta dialéctica no es en ningún caso platónica, ni hegeliana. Es mucho más heraclítea, mucho más polémica (pólemos). Sospechamos que lo implícito en esta noción de dialéctica es un intento por soslayar la noción de superación (Aufhebung) tan persistente en la comprensión hegeliana. Esto es claro en las reflexiones de Kusch, particularmente en su texto El pensamiento popular desde el punto de vista filosófico (consideraciones sobre el método, los supuestos y los contenidos posibles) donde destaca la referencia a una "simple tensión dialéctica":

Entonces, en vez de consistir la dialéctica en una superación cerrada a lo largo de un camino constatado, ella se mantiene abierta en algo que la condiciona, y que llamaríamos la simple tensión dialéctica. Esta tiende no a una superación, sino a una mediación que no sigue un sendero previsto o logos, sino que apunta al centro de las oposiciones en general $(3,540)$.

Esta indicación es muy importante porque enlaza no solo su conocimiento de la tradición filosófica occidental, sino la propia exigencia de posicionar una perspectiva sobre la tensión y la relación de los contrarios evitando una lectura teleológica y progresiva ("sendero previsto o logos"), privilegiando la mediación a partir de "otro sendero" que no sigue solamente la "síntesis", sino que se concentra en las "oposiciones".

Es posible comprender esta "simple tensión dialéctica" desde algunos conceptos fundamentales en su pensamiento tales como el hedor y la pulcritud. Metáforas y figuras tales como la irracionalidad, la pobreza, el miedo, la ira, la invalidez, la marginalidad, entre varias otras, permiten a Kusch posicionar una dicotomía que expresa y explaya un dilema de carácter ontológico-identitario. El hedor impregna lo irracional, la pobreza, el miedo, la ira, la invalidez y la marginalidad de lo americano. El hedor presenta un asunto que Kusch identifica inicialmente con lo popular y que deriva en lo indígena como ejemplo paradigmático (Kusch, El pensamiento 2, 263-273). Por el contrario, la pulcritud participa de la tensión otorgando una contracara impugnable, un ideal que no se alcanza, una búsqueda externa, foránea. La pulcritud funciona como la metáfora de un ser racional, civilizado, mercantil; un ser que domina su voluntad y transforma su mundo (Kusch, América profunda 2, 125-177).

Sin embargo, hedor y pulcritud son parte de un entrelazado algo más complejo. Aquí se posiciona el problema central en la reflexión ontológica de Kusch, a saber, la relación entre el ser y el estar en tanto que condición de posibilidad para la comprensión de su pensamiento. Indica Kusch en El pensamiento indígena y popular 
en América: "Ya en este punto se reanuda la refriega secular de América, porque vuelve a enfrentarse con toda evidencia estar y ser, pueblo y minoría, lo pardo y lo blanco" $(2,251)$. Prosigue Kusch acerca del problema del ser y el estar en Esbozo de una antropología americana:

Aquí la reflexión asume un papel importante, invierte el sentido de la voluntad de ser, para descubrir que lo fundante no es el es, sino lo dado que está. Es una dimensión que la reflexión debió explorar alguna vez pero que nunca lo ha hecho.

Entonces, ¿qué es eso de estar? No es transferible a la tradición. Tampoco se refiere a la caída abstracta, ni el problema aquí es cómo se digita la redacción de una constitución o el descubrimiento experimental de leyes físicas, porque todo esto pertenece a otros aspectos de lo que es. Indagar esto es hacer una reflexión en el sentido de reflejarse radicalmente. Invierte la voluntad de ser, para descubrir que lo fundado no se refiere al ser, sino a lo dado que está. Se trata en el fondo del problema del fundamento. El requerimiento del fundamento exige que lo encontremos a este en algo ajeno al es, en cierta medida en lo otro, que está (3, 389-390).

En consecuencia, el problema del ser y el estar se "juega" bajo el entendimiento de un momento fundamental que no "es el juego" sino que necesariamente "está en juego" y que "invierte la voluntad de ser" hacia la "facticidad del estar". Esto nos permite comprender el lugar del estar como fundamento filosófico, como punto de partida para su pensamiento. Siguiendo esta línea Kusch cuestiona la paradoja occidental del ser y el no-ser; estableciendo su concepto fundamental de "estar-siendo", que apela a un tercer modo de comprensión abierto a la problematización de la autenticidad de la experiencia en el mundo americano. Esto es claro en Geocultura del hombre americano donde sostiene:

Y he aquí nuestra paradoja existencial. Nuestra autenticidad no radica en lo que Occidente considera auténtica, sino en desenvolver la estructura inversa a dicha autenticidad, en la forma de "estar-siendo" como única posibilidad. Se trata de otra forma de esencialización, a partir de un horizonte propio. Solo el reconocimiento de este último dará nuestra autenticidad $(3,239)$.

El concepto de "estar-siendo" - a modo de "resolución de la simple tensión dialéctica" mediante la concentración en las oposiciones- muestra un acercamiento hacia aquello que nombra como la "esencialización" en términos más bien "existenciarios". Respecto de este punto es impresionante la funcionalización (incluso metodológica) de varias reflexiones de Heidegger (verbigracia, el ser-ahí, el ser-en-elmundo, el estado-de-resuelto, la angustia, la autenticidad, entre otros). Podemos decir que Kusch lleva a cabo una "analítica existenciaria" (Heidegger, Ser y Tiempo, 53233) sobre lo popular y lo indígena, constituyendo a un hallazgo o bien un hito en su propuesta filosófica. Así también, la constante indicación hacia una existencia con 
una calidad particular, calificada con adjetivos tales como hedienta o pulcra; india, parda o blanca; popular, marginal o burguesa, tensiona una noción de existencia desde su posición o su "lugar de reflexión" (Kusch, Esbozo 3, 381-391). La localización del pensar es probablemente otro de los ejes en su filosofia. Para Kusch el sentido del "lugar" de reflexión es inicialmente establecido por la "aproximación geocultural" (Kusch, Geocultura 3, 89-111), que se relaciona ulteriormente con los modos de pensamiento y de existencia que acontecen y han acontecido de forma diversa "en estas tierras". Kusch desarrolla un sendero para el pensar enlazado con el paisaje en el que se presenta. Son decidoras, en esta perspectiva, sus indicaciones acerca de la ciudad y su periferia (Kusch, Mala vida 1, 375-390), o bien de las montañas y la puna (Kusch, Indios 1, 149-234), asuntos que rodean de forma gravitante toda su obra.

En este contexto, y debido a la complejidad de un acercamiento a su pensamiento, dedicaremos las siguientes páginas a retomar y profundizar en algunos aspectos fundamentales de su filosofía mediante la descripción de tres enfoques. Esto implica considerar tres niveles o dimensiones de análisis que, de cualquier modo, funcionan de forma conjunta y co-perteneciente en su obra. En consecuencia, desplegaremos brevemente tres enfoques que procuran entrelazar el pensamiento de Kusch: el enfoque fenomenológico-cultural, el antropológico-simbólico y el filosóficopolítico. Estos enfoques buscan una entrada propedéutica que persigue la articulación de un cuestionamiento en directa relación con las oportunidades de un planteamiento filosófico que se construye conjuntamente desde la reflexión sobre un método y la problematización de la noción de lo popular y de lo indígena en América Latina.

\section{ELENFOQUE FENOMENOLÓGICO-CULTURAL}

Es preciso establecer la influencia de la fenomenología en la reflexión filosófica de Kusch. La atención a los modelos de comprensión e interpretación del mundo de la vida serán elementos funcionales a sus propuestas durante toda su obra ${ }^{6}$. Probablemente podríamos hablar de una conversación constante con Husserl y Heidegger, entre otros autores, y con elementos conceptuales tales como conciencia, mundo de la vida, ser-en-el-mundo, ser-ahí, acontecer, autenticidad, entre muchos otros. Nos referimos a una conversación constante en la que las reflexiones de Kusch

\footnotetext{
${ }^{6}$ En un modo general podemos sostener que la fenomenología llega a América Latina durante la primera mitad del siglo XX. México y Argentina son lugares privilegiados en la recepción de estas ideas por medio de figuras tales como Alfonso Reyes, José Vasconcelos, José Gaos, en México, y Alejandro Korn, Coriolano Alberini, Carlos Astrada, Luis Juan Guerrero, Francisco Romero, José Luis Romero, en Argentina. Muy Importante fue la influencia de José Ortega y Gasset en la circulación de las obras de Husserl, Scheler, Hartmann, Heidegger, entre otros, y en la divulgación de esta corriente filosófica que dominó la reflexión continental europea. La apropiación de la fenomenología en México (Pereda; Zirión Quijano) y Argentina (Jalif de Bertranou) expresa el alcance e influencia de un pensamiento que se opone al positivismo (corriente hegemónica en América Latina durante el siglo XIX). Sostenemos que Rodolfo Kusch puede ser relacionado directamente a la fenomenología, principalmente en la construcción de su método.
} 
se establecen de forma crítica, apropiativa y resignificante. Apunta Kusch concerniente a la fenomenología en El pensamiento popular desde el punto de vista filosófico (consideraciones sobre el método, los supuestos y los contenidos posibles):

La fenomenología disuelve la oposición entre sujeto y objeto para restaurar lo mismo, y este se refiere al modo propio de ver, o, en el fondo, de existir. Entre nosotros la fenomenología debería incorporar entonces este modo propio de existir, y para consolidar este se impone el análisis del pensamiento popular, tomando a este como hipótesis de trabajo, aun cuando es un modo de lo mismo de nuestro pensar, y por consiguiente no puede, si no, referir al pensar en general $(3,500)$.

Bajo este enfoque resulta necesario identificar por lo menos dos niveles de influencia de la fenomenología en el pensamiento de Kusch. Por una parte, la necesidad de una comprensión que se ubica en tensión con la dicotomía moderna entre el sujeto y el objeto y, por otra, la entrada a lo popular como hipótesis de trabajo y como articulación propia del pensar en general. Ambos niveles son radicales en sus alcances. En este sentido, la ruptura con la filosofia moderna del sujeto se explica fenomenológicamente y exige focalizaciones discursivas importantes. Este enlace permite a Kusch direccionar el pensar filosófico hacia componentes culturales (dentro de los cuales destaca la cultura popular, y por cierto, lo indígena).

Este giro cultural es probablemente unos de sus aportes centrales al pensamiento filosófico americano. Kusch nos obliga a volver sobre lo cultural en un cuestionamiento por el pensar y la existencia. Esto deriva en la integración ontológica-existenciaria de la cultura y de las modulaciones que la configuran. Aquí lo cultural funciona como una conceptualización que canaliza sus propuestas fundamentales desde la perspectiva fenomenológica. Esto es claro cuando refiere a la relación fundamental entre cultura y suelo, conducente a una comprensión paradigmática dentro de su pensamiento. Indica respecto de ello Rodolfo Kusch en Geocultura del hombre americano:

Y ese suelo así enunciado, que no es ni cosa, ni se toca, pero que pesa, es la única respuesta cuando se hace la pregunta por la cultura. Él simboliza el margen de arraigo que toda cultura debe tener. Es por eso que uno pertenece a una cultura y recurre a ella en los momentos críticos para arraigarse y sentir que está con una parte de su ser prendido al suelo. Uno piensa entonces qué sentido tiene toda esa pretendida universalidad enunciada por los que no entienden el problema. No hay otra universalidad que esta condición de estar caído en el suelo, aunque se trate del altiplano o de la selva. De ahí el arraigo y, peor que eso, la necesidad de ese arraigo, porque, si no, no tiene sentido la vida. Es la gran paradoja de la cultura. Si por un lado es la más cruel de las revoluciones porque nos desnuda totalmente (pensemos en la desnudez de Van Gogh), por el otro es el definitivo domicilio en el mundo, como que tiene por misión una nueva creación del mundo. Realmente no deberíamos entender las transformaciones, sino en este único sentido que brinda la cultura, como algo que apunta nada más que a mi vida aquí y ahora $(3,110)$. 
Vemos claramente en la cita la urgencia de congregar lo cultural con lo filosófico sin reducciones ni atomizaciones. Es evidente la profundidad de la noción de suelo en su comprensión de la cultura, ya que la pregunta por la existencia se aborda y problematiza en el sentido de pertenencia o domicilio, en el arraigo -en un claro diálogo con Heidegger y su concepto de suelo (Boden $)^{7}$-. La cultura expuesta desde esta matriz evita tanto las lecturas biologicistas y folklóricas como las lecturas teleológicas o progresivas. En cierto sentido las evade en favor de un sendero donde ambos elementos "cultura" y "suelo" funcionan existenciariamente ("a mi vida aquí y ahora").

\section{ELENFOQUE ANTROPOLÓGICO-SIMBÓLICO}

Identificamos a la antropología como una ciencia social con la que Kusch dialoga fructíferamente, y donde despliega una importante referencia a lo simbólico. Aquello que nombramos como el enfoque antropológico-simbólico busca consolidar una perspectiva ligada a otro tempo, distinto pero relacionado al fenomenológicocultural. La contribución de la antropología (y por cierto de la etnografía) a la obra de Kusch puede situarse como parte de una reflexión atenta a la complejidad del fenómeno cultural americano. Sobre ello, rescatamos algunos elementos significativos directamente relacionados con el ejercicio de un pensar sobre lo americano desde lo experiencial. Sostiene Rodolfo Kusch en América profunda:

Pero no hay labor más eficaz, para dar solidez a esta búsqueda de lo americano, que la del viaje y la investigación en el mismo terreno. Desde un primer momento pensé que no se trataba de hurgarlo todo en el gabinete, sino recoger el material viviente en las andanzas por las tierras de América, y comer junto a su gente, participar de sus fiestas y sondear su pasado en los yacimientos arqueológicos; y también debía tomar en cuenta ese pensar natural que se recoge en las calles y en los barrios de la gran ciudad. Solo así se gana firmeza en la difícil tarea de asegurar un fundamento para pensar lo americano $(2,5)$.

No podemos soslayar las intensas horas de trabajo de observación y recopilación de materiales "fonomagnéticos y fotográficos" (Kusch, Observaciones 4, 73-118). Estos materiales son evidencia de horas de comprensión y convivencia en las comunidades, horas de silencio en la puna, finalmente, constitutivas de una reflexión filosófica y vivencial que no se aleja del lugar desde el que se ejecuta. Acerca de este

\footnotetext{
${ }^{7}$ La relación con Heidegger es consecuencia de una profunda lectura que deriva en la problematización de varias de sus principales propuestas filosóficas. Ciertamente, Kusch estudia sus escritos durante su formación filosófica en la Universidad de Buenos Aires (en 1948 obtiene el título de Profesor de Filosofia) y los utiliza e interpreta abiertamente en toda su obra. Entre los diversos problemas filosóficos destacamos la lectura de los problemas relativos al proyecto de destrucción de la historia de la metafísica (Heidegger, Ser y Tiempo, 6, 30-37), pero también -y particularmente- su acercamiento a la noción de suelo (Boden) en términos de arraigo (Heidegger, ¿Qué es metafisica?, 65-92; Filosofia, ciencia y técnica, 207-232). Arraigo que se problematiza por medio de un acercamiento "geocultural", capaz de reconocer su especificidad "en estas tierras" como un elemento significativo de diferencia e identidad.
} 
punto es necesario evitar la identificación del trabajo de campo de Kusch con un mero registro participativo. Por el contrario, implica comprender e interpretar los fenómenos culturales desde la experiencia, volviéndola parte de su reflexión filosófica a partir del trabajo de campo. Sobre ello, indica Kusch en Geocultura del hombre americano:

Desde el punto de vista del trabajo de campo, evidentemente esta última faz hace que en virtud del tipo de trabajo antropológico el observado deja de ser mero objeto y se convierte en sujeto, dado que se refiere a algo existente. A su vez, en tanto es existente tiene un proyecto o posibilidad de ser. [...] Pero en tanto esto ocurre, la relación entre el investigador y el investigado, o sea, entre el observador y el observado, que era de sujeto a objeto, se convierte en una relación sujeto a sujeto $(3,210)$.

Reconocemos en la cita la notoria necesidad de relevar un activo diálogo con la teoría antropológica, que podemos direccionar hacia la antropología simbólica ${ }^{8}$. La antropología de Kusch es siempre filosófica y en tanto que filosófica retoma, utiliza y examina al símbolo y los horizontes simbólicos, toda vez que pregunta por los fenómenos culturales. Es decir, el enfoque antropológico en el pensamiento de Kusch recurre al potencial del símbolo en el tratamiento e interpretación del fenómeno cultural. Una entrada hacia su noción del símbolo y de lo simbólico es la indicada por Kusch en un breve ensayo titulado El problema del símbolo:

Pero no se trata del símbolo solo como cosa puesta ante la vista, porque sería mera imagen, sino como cosa que sobrelleva y resuelve una tensión. Es que el símbolo refleja lo mismo del sujeto aunque puesto ante la vista. Sobrelleva la tensión del sujeto, debida a su desconstitución, pero figurada a través de oposiciones. Según esto, el símbolo se da en el margen de lo objetual y es lo mismo que el sujeto, lindando con las cosas y presentando a través del juego de oposiciones la tensión del sujeto.

En tanto símbolo es la culminación ceremonial de un sujeto que compensa su indigencia, aquel se desempeña en un espacio dramático, que reitera lo mismo del sujeto, con la misma tensión lograda a través de las oposiciones. Las oposiciones fingen o figuran la tensión en tanto es lo puesto ante la vista de lo mismo que el sujeto $(3,490)$.

En la cita se despliega el sentido del símbolo en torno a la desconstitución del sujeto desde su "indigencia". En la "indigencia" presenta una metáfora que involucra y propone una salida a la dicotomía moderna entre el sujeto y el objeto. El símbolo

\footnotetext{
${ }^{8}$ El diálogo entre la antropología simbólica y Kusch no se cierra en el acceso a la cultura como un estudio de los símbolos y el significado que tienen en cada una, o bien en cómo dichos símbolos se han mantenido y transmitido en tanto que una construcción cultural de la realidad por medio de las diferentes manifestaciones simbólicas que esta configura (Burke, 125-154). Para Kusch el símbolo en tanto que arquetipo pertenece a la humanidad, no obstante su sentido pertenece y se vectoriza desde una cultura particular (Kusch, El problema $3,489-495)$.
} 
canaliza la indigencia del sujeto, que en su "desconstitución" se devela como "tensión del sujeto". El problema del símbolo contiene la posibilidad de comprensión de ciertos elementos culturales que contienen un potencial de interpretación fronterizo y dificultoso que re-estructura la dicotomía "sujeto/objeto", desde la tensión y las oposiciones. Finalmente, lo que buscamos establecer desde un enfoque antropológicosimbólico funcional a la comprensión de la obra de Kusch es un vehículo de análisis e interpretación complementario. Este enfoque profundiza y radicaliza aspectos que, en una dimensión y profundidad simbólica, acarrean contenidos significativos; contenidos que son plausibles de rastrear en su obra, principalmente relacionados con cómo comprender lo popular y lo indígena en términos de sus producciones y prácticas simbólicas.

\section{EL ENFOQUE FILOSÓFICO-POLÍTICO}

Debemos insistir en el consciente esfuerzo de Kusch por reconducir un nivel de análisis e interpretación que podemos considerar como filosófico-político. Bien sabemos que la filosofía puede referirse a cuestionamientos políticos, a saber, a las formas de gobierno y sus condiciones de legitimidad y, por cierto, a problemas relacionados con los derechos, la libertad y la justicia. Ahora bien, la política también puede comprenderse como una actitud reflexiva y existencial atenta a las relaciones que se presentan entre la actividad política y el mundo que esta configura (Ferrater Mora). Al alero de estos problemas proponemos incluir el enfoque filosófico-político en la obra de Kusch como un elemento inseparable de su pensamiento y plausible de ser considerado como una arista complementaria en su proyecto intelectual. El grado de comprensión axiológica y la consistente evidencia biográfica de su compromiso con algunas organizaciones y sus propuestas políticas (peronismo de izquierda, sindicalismo, entre otras) nos permiten reconocer una perspectiva en la que la noción de lo popular y de lo indígena canalizan una reflexión concerniente a la actividad política que busca cultivar un mundo. En Geocultura del hombre americano Kusch señala este asunto realizando un enlace decisivo entre cultura y política:

Ahora bien, si la cultura es estrategia para vivir en un lugar y en un tiempo entonces también es política.

Pero una política en este sentido, en un continente como el nuestro no puede ser política digitada, tecnificada, como son las fórmulas a las que estamos acostumbrados. Ha de ser político en su sentido profundo como algo que consiste en despertar un ethos $(3,156)$.

En la cita Kusch despliega un problema central relativo a la condición política y cultural en América como un espacio en juego, en apertura. Hay una profunda crítica donde destacan sus indicaciones referidas a un ethos dormido, a un sentido político profundo que consolide una decisión política y cultural americana en camino 
al encuentro de su autenticidad. Al parecer nuestro autor intuye la inherente problematicidad de la construcción identitaria realizada en estas tierras por las llamadas "republiquetas sudamericanas" (Kusch, América 2, 251). Es preciso reconocer que varias de sus indicaciones en torno a la construcción de la nación latinoamericana se dirigen a desestabilizar la teleología y el progresismo que ahonda en los proyectos desarrollistas latinoamericanos (Kusch, Seminario 4, 459-466).

Siguiendo este camino un enfoque filosófico-político pretende, de manera consecuente, reconocer el talante de un pensamiento que no se desliga de su realidad concreta, que atiende y comprende las inconsistencias de no pensar "con" el indígena y "con" lo popular. Este diagnóstico se encuentra en su noción del "hedor". Porque finalmente para Kusch el hedor de lo americano tiene y proyecta un rendimiento político en cuanto que configura un vehículo para reflexionar respecto del creciente relativismo identitario y el consecuente vacío y futilidad en la construcción de un ideario nacional, que a su vez adolece de tener un sí mismo oculto, cerrado, separado, amputado (Kusch, América 2, 248-254). En suma, cada vez que Kusch utiliza el concepto de "hedor" nos provoca y conduce a una reflexión, no solamente en términos existenciales sino que también en términos filosófico-políticos, que cuestiona los cimientos de toda construcción identitaria de lo indígena y lo popular, y que apunta a una tensión con el proyecto civilizatorio desarrollista latinoamericano. El enfoque filosófico-político en el pensamiento filosófico de Kusch nos dirige a problematizar y profundizar en el "hedor", todo aquello olvidado, amputado, marginado, para encontrar su "aroma".

\section{EL MÉTODO, LO POPULAR Y LO INDÍGENA COMO HORIZONTES DE PREGUNTA}

Deseamos complementar nuestras indicaciones anteriores volviendo sobre algunos tópicos convergentes en el pensamiento de Kusch, a saber, el método, lo popular y lo indígena en tanto que horizontes de pregunta en su filosofia. Procuramos identificar el método como plataforma explicativa del asunto central para su pensamiento filosófico, considerando el lugar donde Kusch lo aplica y fortalece, es decir, inicialmente en el mundo popular y marginal de Buenos Aires, y luego en aquel mundo andino que atraviesa junto con lo indígena su obra madura. En este contexto, enfrentaremos el problema del método considerando los enfoques y las reflexiones ya indicadas. Queremos reconocer en el método de Kusch un modo de acercamiento, de análisis e interpretación, que estructura una plataforma de comprensión para los fenómenos culturales americanos de modo existenciario. En este sentido, indica Kusch asertivamente acerca del método en $E l$ pensamiento popular desde el punto de vista filosófico:

Método supone la descripción de cómo se recorre el camino. Pero en el operar mismo no hay modo, sino apuestas en el sentido de Ricoeur, a partir de un campo de objetividad surgido a medias de los símbolos populares. Se apuesta al logro de la índole de lo otro a partir del operar mismo $(3,502)$. 
El cómo que nos propone Kusch está enfrentado a la inmovilidad de una sustancia o "quintaesencia". El método es la operatividad que se establece como un recorrido, como un camino en realización. Un recorrido como "apuesta"; una apuesta en tanto que apertura a los presupuestos que tenemos hacia lo desconocido. Luego, es aquello desconocido que ronda en lo simbólico, en la medida en que se comprende desde su acontecer cambiante, siguiendo a Kusch desde su "operar mismo". En este punto, es necesario introducir el concepto fundamental de fagocitación como un pivote teóricometodológico. Sobre ello señala asertivamente Kusch en América profunda:

Pero esta misma oposición, en vez de parecer trágica, tiene una salida y es la que posibilita una interacción dramática, como una especie de dialéctica, que llamaremos más adelante fagocitación. Se trata de la absorción de las pulcras cosas de Occidente por las cosas de América, como a modo de equilibrio y reintegración de lo humano en estas tierras.

La fagocitación se da por el hecho mismo de haber calificado como hedientas a las cosas de América. Y eso se debe a una especie de verdad universal que expresa, que, todo lo que se da en estado puro, es falso y debe ser contaminado por su opuesto. Es la razón por la cual la vida termina en muerte, lo blanco en lo negro y el día en la noche. Y eso es sabiduría y más aún, sabiduría de América (2, 18-19).

Con la noción de fagocitación, es decir, la absorción de lo occidental en favor del equilibrio y la reintegración, Kusch construye una dimensión crítica de análisis relativa a una interacción dramática y crucial, ulteriormente, focalizada en la (re)construcción de los procesos culturales e identitarios que han acontecido en América. Esta interacción persigue el acontecer histórico en la facticidad de una dialéctica centrada en los opuestos, capaz de constituir una sabiduría americana. En consecuencia, es posible reconocer a la noción de fagocitación como un concepto fundacional que permite comprender al pensamiento de Kusch desde la complejidad de horizontes de pregunta tales como el método, lo popular y lo indígena. La fagocitación contiene elementos analíticos provenientes de una experiencia (y una convivencia) con lo popular, sirviéndose de estos como vehículos para filosofar de lo urbano, lo mestizo, lo argentino y, por cierto, de lo latinoamericano. Por otra parte, lo indígena es siempre un problema abierto. En lo indígena se presenta lo simple, lo arcaico y lo antiguo, lo seminal; lo indígena en América exhibe un axioma fundamental en cuanto que reconoce la existencia de un pensamiento filosófico indígena como trasfondo del pensar americano. Aquella reflexión arcaica, seminal, indígena, se enfrenta inexorablemente a la aculturación (Kusch, América 2, 179-215), y para ello utiliza a la fagocitación (de lo occidental) como respuesta del sujeto cultural americano. Esta respuesta es fundacional

\footnotetext{
${ }^{9}$ Paul Ricoeur sostiene en Finitud y culpabilidad: “Tal es nuestra única apuesta. Únicamente puede ofenderse por este modo de enfocar la investigación el que crea que la filosofía solo puede salvaguardar su iniciativa y su autonomía a condición de eliminar todo presupuesto previo. Cualquier filosofía que arranque en el pleno lenguaje es una filosofia que cuenta por el mismo hecho con algún presupuesto previo. Lo que le corresponde hacer para salvar su honradez es explicitar y aclarar sus presupuestos, enunciarlos como creencias, elaborar las creencias como en apuestas e intentar ganar la apuesta transformándola en comprensión" (498).
} 
porque demuestra los modos de supervivencia de lo popular y lo indígena. Frente a la aculturación el ejercicio de fagocitación soslaya (y subvierte) la imposición cultural. Es un ejercicio de resistencia y conservación, de adaptación y acomodo, ulteriormente, es un ejercicio creador, un ejercicio vital. Señala Kusch acerca de este asunto en Esbozo de una antropología filosófica americana:

El término vida no se define, sino que transita entre el estar y el es. Constituye el término central de una conciencia natural. Y en tanto que media entre lo puramente dado que está, y lo determinable del es, traza ahí la parábola de la biografia, no solo propia, sino también la de las piedras, con los episodios que se van dando, pero que siempre pudieron haber sido otros, y que únicamente es corregida con la posibilidad y no la efectivización de un "saber vivir" $(3,403)$.

La vida como problema en la filosofia de Kusch se resuelve con el transitar entre el estar y ser que tiende (no progresivamente) hacia un "saber vivir". La vida y su sabiduría funcionan como mediación, como una vía simbólica para comprender el "estar viviendo". La vida es dinámica y transitoria, constituye un juego, un vaivén, en el que la noción de fagocitación integra un carácter lúdico, de mediación e interoperación. La fagocitación en términos de condensación conceptual de sus propuestas metodológicas (en un sentido profundo) nos invita a detener nuestra mirada en aquello que lo popular o lo indígena desarrollan y vectorizan, finalmente, como "sabiduría de vida", como un "saber vivir". La fagocitación se comprende desde el "saber vivir" porque es algo dinámico, que se regenera y acontece con la seriedad de un juego que involucra la vida. Advierte sobre el juego: "Lo lúdico abre un área de interpretación de lo humano que no es fija sino dinámica. A partir de aquí no tiene sentido trazar modelos rígidos, sino en todo caso en movimiento" (Kusch, Esbozo 3, 410). Fagocitar es entonces desocultar este carácter lúdico, dinámico, abierto a la experiencia que configuran la vida y lo vital, y que presenta en lo popular y lo indígena dos vectores fundacionales que avizoran una comprensión del mundo de la vida como horizontes de pregunta sobre lo americano.

Finalmente, Kusch no se agota, no se concluye ni se cierra. Hemos intentado reconocer su calidad reflexiva mediante la descripción y la profundización de un paisaje conceptual provisto de entradas múltiples en la ardua labor de comprender e interpretar su pensamiento. La convergencia que nos proponen los tres enfoques junto con la tríada conceptual relativa al método, lo popular y lo indígena en tanto que "horizontes de pregunta" envuelven un ejercicio de re-elaboración y actualización de propuestas sustantivas, que re-direccionan el sentido de la filosofia desde y sobre América.

Universidad de Chile*

Facultad de Filosofia y Humanidades

Centro de Estudios Culturales Latinoamericanos Avda. Ignacio Carrera Pinto \# 1025, Ñuñoa, Santiago (CHILE)

aviveros@u.uchile.cl 


\section{OBRAS CITADAS}

Azcuy, Eduardo (comp.). Kusch y el pensar desde América. Buenos Aires: Fernando García Cambeiro, 1989.

Bordas de Rojas, Nerva. Filosofia a la intemperie. Buenos Aires: Biblios, 1997.

Burke, Peter. ¿Qué es la historia cultural? Barcelona: Paidós, 2006.

Camaly, Mario, Luis Jalfen y Mauricio Langón (eds.). Cuartas Jornadas del Pensamiento Filosófico Argentino. "La obra de Rodolfo Kusch" Homenaje a diez años de su muerte. Buenos Aires: FEPAI, 1993.

Casalla, Mario. "Filosofia y cultura nacional en la situación latinoamericana contemporánea", Revista Nuevo Mundo 1 (1973): 36-50.

Carmagnani, Marcello. El otro Occidente: América Latina desde la invasión europea hasta la globalización. México: Fondo de Cultura Económica, 2004.

— Formación y crisis de un sistema feudal: América Latina del siglo XVI a nuestros días. México: Siglo Veintiuno, 1980.

Cullen, Carlos. "La América Profunda busca su sujeto. De cómo entiende la filosofía Rodolfo Kusch". Espacios de crítica y producción 43 (2010): 88-98.

__ "Ser y estar. Dos horizontes para definir la cultura", Stromata 34.1-2 (1978): 43-52.

Dussel, Enrique, Eduardo Mendieta y Carmen Bohórquez. El pensamiento filosófico latinoamericano, del Caribe y "Latino" (1300-2000): historia, corrientes, temas y filósofos. México: CREFAL-Siglo XXI, 2009.

Echeverría, Bolívar. La modernidad de lo barroco. México: ERA, 1998.

— Las ilusiones de la modernidad. México: El Equilibrista, 1995.

Ette, Ottmar. Viellogische Philologie. Die Literaturen der Welt und das Beispiel einer transarealen peruanischen Literatur. Berlin: Kadmos Verlag, 2013.

Ferrater Mora, José. Diccionario de Filosofia. Barcelona: Ariel, 2004.

Flores, Gustavo. "La filosofía del mito: Rodolfo Kusch y Juan Rulfo". Journal of interdisciplinary literary studies / Cuadernos interdisciplinarios de estudios literarios 3.2 (1991): 179-195.

Fraschini, Alfredo. Hacia un proyecto cultural argentino. El ideario de Rodolfo Kusch como punto de partida para su formulación. Córdoba: Ediciones IAPCH, 2010.

Halperin Donghi, Tulio. Historia contemporánea de América Latina. México: Alianza, 1987.

Heidegger, Martín. ¿Qué es metafisica? Madrid: Alianza, 2009

_ Filosofia, Ciencia y Técnica. Santiago: Universitaria, 2007.

— Ser y Tiempo. Buenos Aires: Fondo de Cultura Económica, 2003.

Husserl, Edmund. Erfahrung und Urteil. Prag: Academia, 1939.

Jalif de Bertranou, Clara Alicia. "Recepción y elaboración de la fenomenología en la Argentina". CUYO Anuario de Filosofia Argentina y Americana 13 (1996): 45-84. 
Kuhn, Helmut. "The phenomenological concept of horizon". Philosophical Essays in Memory of Edmund Husserl. Ed. Marvin Faber. Cambridge: Harvard University Press, 1940: 106-123.

Kusch, Rodolfo. Obras Completas. 4 Vols. Rosario: Fundación Ross, 2009.

—_ Indigenous and popular thinking in América. Durham: Duke University Press, 2010.

Maturo, Graciela. Filosofia y literatura desde América Latina. Manizales: Universidad de Caldas, 2007.

Mignolo, Walter. "Introduction". Rodolfo Kusch. Indigenous and popular thinking in América. Durham: Duke University Press, 2010. xv-liv

Local histories / Global designs: coloniality, subaltern knowledges, and border thinking. Princeton: Princeton University Press, 2000.

Sada, Gabriel. Los caminos americanos de la filosofia de Rodolfo Kusch. Buenos Aires: Fernando García Cambeiro, 1996.

Montes, Jaime. "La filosofia americanista de Rodolfo Kusch: el hombre americano entre el 'ser alguien' y el 'estar no más"'. Revista de Filosofia 45-46 (1995): 65-70.

Pagano Fernández, Carlos María. Un modelo de filosofia intercultural: Rodolfo Kusch (1922-1979). Aproximación a la obra del pensador argentino. Aachen: Concordia Monographien, 1999.

Pereda, Carlos. La filosofia en México en el siglo XX. Apuntes de un participante. México: CONACULTA, 2013.

Reigadas, María. "Filosofía y ciencias del hombre en América Latina. Homenaje a Rodolfo Kusch". Megafón. Revista interdisciplinaria de Estudios Latinoamericanos 17-18 (1986): 19-27.

Ricoeur, Paul. Finitud y culpabilidad. Madrid: Taurus, 1969.

Rivera Cusicanqui, Silvia y Barragán, Rossana, eds. Debates postcoloniales: una introducción a los estudios de la subalternidad. La Paz: SEPHIS-Aruwiyiri, 1997.

Rubinelli, María Luisa. Reflexiones actuales sobre el pensamiento de Rodolfo Kusch. San Salvador de Jujuy: Editorial Universidad Nacional de Jujuy, 2001

Scannone, Juan Carlos. Sabiduría popular, símbolo y filosofia. Diálogo internacional en torno a una interpretación latinoamericana. Buenos Aires: Guadalupe, 1984.

Steffen, Guillermo. Estar en América y el encuentro con el otro: una psicología válida para los sudamericanos fundamentada sobre el pensamiento filosófico de Rodolfo Kusch. Buenos Aires: Centro de Estudios Latinoamericanos, 1990.

Toribio, Daniel. La lógica de la negación en el pensamiento de Rodolfo Kusch. Buenos Aires: Fondo Nacional de las Artes, 1984

Unión del Personal Civil de la Nación (UPCN). Octubre Nacional. Buenos Aires: UPCN, 2006.

Universidad Santo Tomás. Análisis. Revista Colombiana de Humanidades 77 (2010): 25-178.

Vasconcelos, José. La raza cósmica: misión de la raza americana. Notas de viaje a la América del Sur. Madrid: Agencia Mundial de Librería, 1925. 
Alejandro Viveros Espinosa

VOX. Diccionario Latín-Español / Español-Latín. Barcelona: Bibliograf, 2001.

Zirión Quijano, Antonio. La fenomenología en México. Historia y Antología. México: Universidad Nacional Autónoma de México, 2009.

_ Historia de la fenomenología en México. Morelia: Red Utopía / Jitanjáfora Morelia, 2003. 